\title{
CONFERENCES AND SYMPOSIA
}

\section{Report on the Eighth International Reflectometry Workshop (IRW8) held at St.Petersburg, Russia 2-4 May 2007}

\author{
G.D.Conway \\ Max-Planck-Institut für Plasmaphysik, EURATOM-Association IPP, Garching, \\ D-85748, Germany
}

Submitted to: Nucl. Fusion

PACS numbers: 52.70.Gw, 52.35.Ra

\section{Introduction}

The International Reflectometry Workshop for Fusion Plasma Diagnostics is the biennial forum for the fusion reflectometer community to present the latest results and developments on microwave and millimetre-wave techniques. The eighth workshop (IRW8) - organized in cooperation with the International Atomic Energy Agency - was held at the Ioffe Physico-Technical Institute, St.Petersburg, Russia between May 2nd and 4th, 2007. The workshop was attended by some 47 specialists from around the world; 22 from the EU (France, Germany, Portugal, Spain, UK, Italy, Czech Rep.), 19 from the Russian Federation, 2 from Japan, 2 from Korea, 1 from Brazil and 1 representing ITER. There were, unfortunately, no participants from the USA. A total of 34 oral papers were presented in 6 consecutive sessions - two sessions on density fluctuation measurements, Doppler reflectometry for rotation measurements, theory and simulations, density profile measurements and hardware developments. Following the precedent set at the last meeting in 2005 the workshop proceedings, consisting of short formal papers, were published electronically [1]. Summaries of the second [2], third [3], fifth [4] and seventh workshops [5] are also available.

Reflectometry, as a fusion plasma diagnostic, involves the reflection of a low power (few $\mathrm{mW}$ ) microwave or mm-wave beam from a cutoff layer where the plasma refractive index $N$ approaches zero. For propagation perpendicular to the magnetic field $B$ this occurs when probing frequency equals the plasma frequency $\omega_{o}=\omega_{p e}$ for O-mode $(E \| B)$ or $\omega_{o}^{2}=\omega_{p e}^{2} \pm \omega_{o} \omega_{c e}$ for X-mode $(E \perp B)$ polarization. By measuring the propagation delay or phase shift in the reflected beam as a function of $\omega_{o}$ the position of the cutoff layer (and hence the radial density profile) can be determined. Likewise, information on the density fluctuations around the cutoff position can be obtained from fluctuations in the reflected signal phase and amplitude. 


\section{Density fluctuation and MHD measurements}

Continuing the recent trend density fluctuation and turbulence measurements still dominate the field. Several new results were presented using the standard fixed probing frequency technique. S.da Graca (CFN, Portugal) reported measurements of Toroidal Alfvén Eigenmodes (TAEs) and Alfvén cascades in the ASDEX Upgrade tokamak (AUG) using Q and V-band (33-75 GHz) stepped frequency O-mode reflectometers. By cross-correlating the reflectometer signal at each frequency step with a reference Mirnov coil signal the radial eigenfunction (amplitude and sign) of $n=4 \& 5$ TAEs was obtained showing two antinodes, one located in the edge and one in the core, in good agreement with MHD and gyrokinetic simulations. The maximum TAE amplitude was also estimated at $0.025 \%$ using a $1 \mathrm{D}$ geometric optics model $\delta n / n=(\delta \phi / 4 \pi)\left(\lambda_{o} / L_{n}\right)$ where $L_{n}$ is the density scale length. Global Alfvén Eigenmodes (GAE) were also detected by T.Happel (CIEMAT) in the TJ-II stellarator using a similar frequency hopping system and 1D model. The GAE peaked radially at $\rho \sim 0.75$ with an amplitude $\delta n / n \approx 3 \%$. Alfvén wave (AW) resonances were the topic of a paper by L.Ruchko (Uni. San Paulo). AW with selected mode numbers are excited in the TCABR tokamak using phased ICRF antenna pairs. The AW excite localized density fluctuations at a resonant layer dependent on the local density, the safety factor $q$ and the effective mass $M_{\text {eff }}$. Using a simple homodyne O-mode 16-26 GHz swept frequency reflectometer AW resonances in the edge plasma were detected. Core access, and hopefully a demonstration of $q$ profile and $M_{\text {eff }}$ measurement are planned using an enhanced reflectometer with a $18-72 \mathrm{GHz}$ frequency scan currently under construction. The radial localization of MHD modes was also demonstrated by K.D.Lee (NFRC, Korea) using a novel stepped frequency X-mode reflectometer on the VINETA linear device with a helicon plasma source. Localized low order $m=5$ modes in the plasma edge were found, in good agreement with Langmuir probe measurements.

The behaviour of Geodesic Acoustic Modes (GAM) is currently a popular topic of investigation. GAMs are oscillating poloidal $E \times B$ plasma flows and are closely linked to turbulence and confinement. The long coherence lengths of these modes $(m=0)$ makes them well suited to correlation analysis using poloidally separated reflectometers, such as the O-mode systems on TEXTOR and T-10 tokamaks. A.Krämer-Flecken (FZJülich) presented various results from TEXTOR using correlation time lag fluctuations to show the GAM is a stationary oscillation while the background turbulence rotates. Compared to the low field side mid-plane the associated GAM $\delta n$ perturbation was found to be stronger in the upper poloidal quadrant, consistent with an $m=1$ pressure side-band mode. Radial correlation measurements gave a $\lambda_{r} \approx 5 \mathrm{~cm}$, from which a shearing rate was estimated and found comparable to the turbulence decorrelation time - implying the GAM in TEXTOR has only a weak effect on the turbulence. T10 measurements presented by A.Khamra (Kurchatov) using correlation reflectometers (radial and poloidal) and heavy ion beam probes (HIBP) showed GAM-like behaviour in both $\delta n$ oscillations from the top quadrant $\left( \pm 30^{\circ}\right.$ refl. $)$ and $\delta \phi$ (HIBP). The oscillation 
was largest at the edge, close to the $q=2$ surface (from ASTRA code), in contradiction to TEXTOR where no GAM link to the $q=2$ location was found, just MHD.

\section{Turbulence measurements}

A long standing issue in reflectometry turbulence measurements is the translation of the phase signal $\delta \phi$ to an absolute density fluctuation level $\delta n / n$. The difficulty lies in the instrument response function, which for traditional fixed frequency probing (constant measurement position) is a two-dimensional (2D) problem involving the radial and transverse turbulence $k$-spectra, amplitude and the reflectometer beam geometry (illuminated spot size and divergence etc.). That is, every reflectometer may respond differently depending on the application. Nevertheless, 1D approximations have been shown to give reasonable results. For example, $\delta n / n$ profiles from Tore Supra were shown by R.Sabot (CEA) using two time multiplexed microwave sources in X-mode: Stepped frequency synthesizers for fluctuation and swept frequency VCOs for profile measurements. The $1 \mathrm{D}$ model $\delta n / n=\left(\delta \phi / 2 k_{o}\right)\left(k_{r} / \pi L_{\epsilon}\right)^{1 / 2}$ was employed with fixed $k_{r} \approx 1 \mathrm{~cm}^{-1}$. During beta scaling experiments no variation in the core $\delta n / n \sim 0.5 \%$ was seen, consistent with the weak $\tau_{E}$ confinement degradation. A second channel for radial correlation measurements is currently being commissioned. D.Shelukhin (Kurchatov) also presented O-mode turbulence measurements from T-10 and TEXTOR using a similar 1D model. Attempts were made to characterize and quantify the rising turbulence level and spectral spreading observed with increasing density in ohmic and ECR heated plasmas. Quasi-coherent spectral features were reported extending from the tokamak mid-radius to edge. Overall, the turbulence rose from $\sim 0.5 \%$ in the core to several percent in the edge, with strong poloidal variations suggesting a ballooning nature.

An alternative approach, which avoids the 2D issue, was presented by T.Gerbaud (CEA). Extending the work by colleagues L.Vermare and S.Heuraux the technique combines fast $20 \mu$ s sweep X-mode (V and W-band) reflectometry data from Tore Supra with an iterative $1 \mathrm{D}$ full-wave simulation to estimate $\delta n / n$ radial profiles and $S_{n}\left(k_{r}, r\right)$ spectra. The starting point is the phase fluctuation $\delta \phi(r)=\phi(r)-\langle\phi(r)\rangle$ obtained from IQ detection for each sweep, where the launch frequency $f_{o}$ is mapped to radius $r$ using density profiles (from the same diagnostic) and magnetics, and an average phase computed from a hundred similar sweeps. $\delta \phi(r)$ is segmented and Fourier transformed to give the spectrum $S_{\phi}^{e x p}\left(k_{r}, r\right)$, which is converted to a density fluctuation spectrum $S_{n}\left(k_{r}, r\right)$ via a transfer function $f\left(k_{r}, r\right)=S_{n} / S_{\phi}$ obtained from an iterated 1D full-wave code. Using an initial guess for $S_{n}$ the full-wave code generates a simulated $S_{\phi}\left(k_{r}, r\right)$ from which $f\left(k_{r}, r\right)$ is computed and applied to the $S_{\phi}^{e x p}\left(k_{r}, r\right)$ to create a new input $S_{n}\left(k_{r}, r\right)$. The loop is iterated (5-6 times) until convergence. The $\delta n / n(r)$ profiles (integrated $\left.S_{n}\left(k_{r}, r\right)\right)$ are in good agreement with previous laser scattering measurements for a range of conditions, and consistent with the fixed frequency measurements described above. Using a 1D code is claimed to be sufficient if the data is band-pass filtered to 
remove the high $k_{r}$ components resulting mainly from $2 \mathrm{D}$ effects.

Returning to correlation measurements, T.Happel (CIEMAT) reported constant turbulence radial coherence lengths of $L_{r} \sim 5 \mathrm{~mm}$ across the core region $\rho \sim 0.47-0.74$ of TJ-II by combining stepped frequency measurements and density profiles from the AM reflectometers. Consistent monotonic correlation decays could only be obtained with the complex $A e^{i \phi}$ signal, compared to phase or amplitude alone. V.Vershkov (Kurchatov) used toroidally and poloidally displaced antennas to confirm the long parallel correlation lengths of turbulence in T-10. A strong correlation was observed for high frequency fluctuations along magnetic field lines coinciding with $q$ profile predictions from the ASTRA code. However, low frequency fluctuations showed a lower degree of correlation.

\section{Rotation and $E_{\mathrm{r}}$ measurements - Doppler}

An indication of the continued growth in Doppler reflectometry is that nearly all major tokamak and stellarator devices are now either running or building Doppler systems. The diagnostic principle is generally established: The reflectometer is tilted to the refractive index gradient so that the Bragg back-scattering at the cutoff layer is dominated by the perpendicular fluctuation wavenumber $k_{\perp}=2 N k_{o}$ where $N \approx \sin \theta_{o}$ for flat cutoff layers. The fluctuation movement induces a Doppler shift $f_{D}=u_{\perp} k_{\perp} / 2 \pi$ in the back-scattered signal, where $u_{\perp}=v_{E \times B}+v_{\text {ph }}$ is the turbulence velocity in the plasma $E_{r} \times B$ frame. If the turbulence phase velocity $v_{\mathrm{ph}}$ is known or negligible then the radial electric field $E_{r}$ profile can be extracted. This issue was addressed by G.D.Conway (IPP Garching) who presented $v_{p h}$ simulations from linear and non-linear gyrokinetic codes for AUG H-mode edge and core conditions. In both cases $v_{p h}$ of a few hundred $\mathrm{ms}^{-1}$ were predicted, much less than the electron diamagnetic velocity. For drift-wave, ITG and TEM type turbulence, $v_{E \times B} \gg v_{p h}$ is generally satisfied. For the Bragg component to dominate over the normal specular reflection careful antenna design is a prerequisite. Using simulations the effects of higher Bragg orders and antenna side-lobes were shown as possible explanations for secondary Doppler peaks observed in AUG data.

Several papers were presented on Doppler measurements. From the TJ-II stellarator T.Happel (CIEMAT) discussed the effects of density variation, edge plasma biasing and ECH modulation on the plasma rotation. N.Skvortsova (GPI Moscow) presented observations on the formation of a poloidal velocity radial shear in the L-2M stellarator edge when changing the rotational transform. V.Saenko (GPI Moscow) discussed the statistical characteristics of Doppler fluctuations in L-2M plasmas using a signal increment analysis technique (shift-scale mixtures of normal distributions). The analysis revealed 2 to 3 independent Gaussian processes with different propagation velocities. The behaviour of the processes was seen to change with the application of ECR heating and variations in rotational transform. Although the technique requires further validation it may offer a way of separating various phase velocity components in the Doppler spectrum.

A new attempt at experimental validation of Doppler reflectometry was presented 
by A.Gurchenko (Ioffe) with a comparison against Correlative Doppler Enhanced Scattering (Upper-hybrid resonance back-scatter) and poloidal velocity measurements from spectroscopy of impurity ions in the FT-2 tokamak. The CDES rotation profiles were close to neo-classical estimates of poloidal fluid rotation $\left(2-3 \mathrm{kms}^{-1}\right)$, while the Doppler $u_{\perp}$ values (using analytic values for $k_{\perp}$ ) were smaller, by roughly the ion diamagnetic velocity. Estimates of $v_{E \times B}$ values from spectroscopy and radial force balance were found by V.Bulanin (SPbSPU, Russia) to be in reasonable agreement with $u_{\perp}$ profiles. Any differences were ascribed to the turbulence phase velocity. V.Bulanin also investigated the $u_{\perp}$ reversal across edge during density ramping in FT-2 with lower hybrid heating. A loss of Doppler signal in the high velocity shear region was associated with the suppression of turbulence. A reduction of the edge $u_{\perp}$ profile with the onset of strong MHD was also noted.

The second major application of Doppler reflectometry is the localized measurement of turbulence $k$-spectra. P.Hennequin (LPTP, France) presented comparisons of ohmic and L-mode spectra in Tore Supra from $\mathrm{CO}_{2}$ laser-scattering and Doppler reflectometry with a motorized tiltable GOLA antenna. A change in the spectral index from $k^{-3}$ for $k<10 \mathrm{~cm}^{-1}$ to $k^{-6}$ at higher $k_{\perp}$ was associated with a transition from ITG to TEM and ETG type turbulence predicted by the linear gyrokinetic code KINZERO. Investigations into $\nu^{*}$ and $\rho^{*}$ scaling are planned.

New Doppler systems are also coming online. C.Tröster (IPP Garching) presented details of a new steerable in-vessel antenna currently being commissioned on AUG. Together with a new W-band (75-110 GHz) channel this will allow turbulence $k$ spectrum measurements with a core to edge coverage. N.Oyama (JAEA) described plans to replace the existing X-mode system on JT-60U with a new synthesizer based channel in Q-band and $50 \mathrm{MS} / \mathrm{s}$ real-time data streaming. Using fixed tilt antennas with focusing mirrors the $u_{\perp}$ profile will be measured by changing plasma position to obtain oblique incidence.

\section{Modelling and simulations}

Modelling and numerical simulation studies remain an integral part of the diagnostic development. They aid the interpretation of experimental results, provide diagnostic validation and give insight into the diagnostic response function. Currently the main tool is a time-dependent numerical solution of Maxwell's wave equation in radial and poloidal dimensions (known as 2D full-wave codes) using realistic diagnostic and plasma geometries and simulated fluctuations. Although these codes are computationally intensive they provide the most accurate simulation of the diagnostic behaviour. The main topic of simulation is currently Doppler reflectometry.

Using a 2D O-mode finite-difference time domain (FDTD) full-wave code F.da Silva $(\mathrm{CFN})$ investigated the forward scattering contribution to the Doppler signal. Analytic modelling predicts that multiple forward-scattering along the beam should become relevant at high turbulence levels or long propagation paths, e.g. core probing. Two 
turbulence models were tested: a Gaussian $k$-spectrum with a width $k_{w}<k_{\perp}$ (to suppress backscatter) and a Kolmogrov spectrum with a flat top width $k_{w}>k_{\perp}$. The Gaussian spectrum produced a bursty signal with a Doppler shift $f_{D}$ increasing with the turbulence amplitude. The Kolmogrov spectrum however gave the correct $f_{D}$ independent of the amplitude. Similar results were also obtained by E.Blanco (CIEMAT) using an independent 2D full-wave code. If the probed $k_{\perp} / k_{w}$ is too large then the measured $f_{D}$ is underestimated. The effect becomes worse the higher the divergence of the probing beam. For $k_{\perp}<k_{w}$ the $f_{D}$ is independent of the tilt angle $\theta_{o}$ and turbulence level. These results are important for defining the limits of high $k$ probing. Nevertheless, the ability to recover the $k$ spectrum from Doppler measurements appears to be good. Using a 2D O/X-mode full-wave code with various Kolmogrov $k$ spectral shapes: plateau, inertial and anisotropic (different radial and poloidal widths) and turbulence amplitudes from 1-50\%, C.Lechte (IPF Stuttgart) could reliably reconstruct the input spectra from the Doppler peak power for $\delta n / n$ upto $10 \%$. At high $\delta n / n \sim 50 \%$ the low $k_{\perp}$ part of the measured spectrum is truncated. The reconstruction incorporated a tilt angle dependent calibration factor obtained from single frequency simulations. A Doppler peak amplitude dependence of $A_{D} \propto k_{\perp}^{-0.9}$ was also found by E.Blanco (CIEMAT). The -0.9 index appears independent of $\delta n / n$, despite $A_{D}$ being only linear with $\delta n / n$ upto about $2 \%$, becoming constant above $7 \%$, possibly due to multiple scattering close to cutoff layer. Further work is clearly necessary here to fully understand the geometry factor.

Taking a different approach A.Popov (Ioffe) used theory to investigate the Doppler wavenumber resolution. Modelling the fluctuations as the sum of short range (correlation length) backscattering and long range forward-scattering components, analytic expressions are derived for the scattering efficiencies (using WKB approximation but including beam broadening due to small angle multiple scattering). The scattering efficiencies are found to be sensitive to the probing radial $k_{r}$, meaning the diagnostic spatial resolution depends on the density profile. Nevertheless, the resolution improves if high $k_{r}$ turbulence is suppressed. Investigating analytically the behaviour of radial correlation reflectometry E.Gusakov (Ioffe) presented an algorithm for extracting the turbulence radial wavenumber spectrum from the measured correlation function. A demonstration of the method was shown using UHR backscatter data from the FT-2 tokamak.

Simulation of standard reflectometry using a 2D TLM full-wave code was the topic of a paper by S.Soldatov (FZJ/Kurchatov). Modelling the TEXTOR geometry and reflectometers, the effect of plasma curvature and refraction was shown to reduce the effective spot size (Fresnel zone) and thus broaden the reflectometer poloidal $k_{\theta}$ response. Using a sinusoid perturbation with constant $k_{r}, \delta n / n$ and gradient length $L_{n}$ the complex amplitude response $\sigma_{E}$ falls with increasing $k_{\theta}$, independent of cutoff position and curvature. Reducing $L_{n}$ enhances the $k_{\theta}$ response. 


\section{Density profiles and swept frequency}

The development of swept frequency reflectometers for density profile measurements continues. R.Sabot (CEA) outlined the recent innovations in the X-mode reflectometer systems on Tore Supra. The D-band channel (100-160 GHz) is now operating routinely and complements the $\mathrm{V}$ and $\mathrm{W}$-band channels $(50-110 \mathrm{GHz})$ to give full plasma radius coverage. For low toroidal field operation a Q-band channel (33-50 GHz) replaces the W or D-band. For simultaneous fluctuation measurements a stepped frequency synthesizer source is time multiplexed with the swept sources. The profile reconstruction is fully automatic, with single sweep or burst-mode averaging, and relativistic corrections. An analysis of the profile sensitivity to various measurement error sources was considered. A.Sirinelli (LPTP Palaiseau) presented transport analysis results from the Tore Supra plasma core using the D-band high resolution profile measurements. In ohmic shots the central density peak (so called Mexican hat profile) collapses during sawteeth allowing estimates of heat and particle transport coefficients. Compared to neoclassical values the diffusion coefficient $D$ is found to be much larger while the pinch velocity $V$ is similar in order. With peaked density profiles the low diffusion matches the low fluctuation $\delta n / n \approx 0.1 \%$ observed in the core with fixed frequency reflectometers.

The group delay signal was also used by G.Cunningham (UKAEA) to study ELMs and MHD events in the MAST device. By operating the homodyne $\mathrm{K}, \mathrm{Ka}$ and Uband channels (18-55 GHz with horizontal polarization) with a restricted mini-sweep $(0.9 \mathrm{GHz}$ sweep in $2 \mu \mathrm{s})$ high frequency cutoff layer movements could be resolved with a resolution of a few mm. In addition to discriminating various MHD and ELMs, burst-like events (bulging of the edge density profile) might interpreted as inter-ELM filaments. Coordinated reflectometer and visible camera measurements will help to resolve this in future experiments.

Although the density profile measurement technique is well established, refinements continue to be made. S-H.Seo (KBSI) reported on the novel technique employed with the O-mode reflectometer on the Hanbit mirror device. A single K-band (8-14 GHz) channel is swept in $41 \mu \mathrm{s}$ with heterodyne IQ detection. The relative phase is calculated using an accumulation code then Wavelet analysed. Spurious non-plasma reflections are removed using a "ridge detection" de-noise technique on the spectrogram. Finally, the phase gradient $d \phi / d f$ (group delay) is spline fitted and Abel inverted to give smooth density profiles.

With X-mode probing a precise knowledge of the magnetic field profile is crucial for locating the cutoff layer. L.Fattorini (CFN) reported the effect of $B$ field ripple on profile measurements in $\mathrm{AUG}$, and in particular the corrections required to map the "average" flux surface reconstruction from the CLISTE code (which uses magnetic data from a variety of coils located around the torus) to the antenna toroidal location. After correcting the O-mode vacuum propagation distance and the X-mode initialization the radial error is reduced to 1-2 mm against Thomson Scattering profiles. Generally however, the calculation of meaningful radial error bars from the phase signal is 
problematic. As an alternative, G.Cunningham (UKAEA) argued that a confidence measure (obtained from an extention of the complex demodulation (CDM) method to calculate a running correlation coefficient) can be just as beneficial.

\section{Real-time density profiles}

One area of significant progress in the last couple of years is the development of realtime density profile measurement. This application is of particular importance for ITER plasma position control where reflectometry is planned for measuring plasma-wall gap distances. Two papers were presented describing two different approaches. F.Clairet (CEA) discussed the real-time profile reconstruction on Tore Supra based on their heterodyne $\mathrm{V}$ and W-band (50-110 GHz), X-mode, $20 \mu$ s sweep FM channels, which covers mostly the edge. The core D-band channel is currently not included. Using VME dual processor data acquisition units a single profile consisting of 4 channels of IQ data at $100 \mathrm{MHz}$ sampling with 10 bit resolution can be read-out in $5 \mathrm{~ms}$. The signal analysis is a fast version of the standard inversion implemented in VSIPL vector signal image processing language. With the current $366 \mathrm{MHz}$ CPUs the two stage filtering to remove window and parasitic reflections, the smoothing of the group delay curve on to 100 points, and the profile inversion using the Bottollier interactive algorithm can produce a single profile from each sweep in $50 \mathrm{~ms}$. With faster CPUs it is hoped to improve the speed by a factor of ten. There are also plans to incorporate relativistic corrections using real-time ECE electron temperature data.

The second approach presented by J.Santos (CFN) uses trained neural networks (NN) for the profile inversion stage. The signal analysis procedure was developed for the AUG high and low-field-side O-mode, homodyne, K Ka and Q-band channels. An optimal procedure, involving signal linearization, digital filtering, group delay calculation from sliding FFT, and the NN profile inversion could achieve in roughly $400 \mu \mathrm{s}$ a "burst-averaged" profile of four $20 \mu$ s sweeps with a confidence range of better than $\pm 1 \mathrm{~cm}$. This means, even with current hardware technology, the ITER requirement of unsupervised reliable edge density profiles for plasma position control with a $10 \mathrm{~ms}$ repetition and $1 \mathrm{~cm}$ resolution can be met.

\section{Ultra-fast swept frequency}

Ultra-fast swept frequency reflectometers are seeing a resurgence of interest. T.Tokuzawa (NIFS) described recent upgrades to the ultra short pulse radar on LHD. A new impulse source plus a U-band multiplier have been added to the existing $\mathrm{X} / \mathrm{Ku}$ and Ka-bands (8-60 GHz). 28 channels with a 23 ps pulse and $40 \mu$ s repetition are now operational increasing the reliability of the density profile reconstruction. Currently the U-band is running with 2 test channels, but more will be added in next campaign. Examples of the fine structure of $(1,1)$ MHD islands illustrated the system capability. However, extreme high densities $\left(10^{21} \mathrm{~m}^{-3}\right.$ obtained during pellet injection) are pushing 
the development of a $\mathrm{THz}$ pulsed radar reflectometer using a $800 \mathrm{~nm}$ wavelength femtosecond laser and photoconductive antennas (50 fs pulse, $80 \mathrm{MHz}$ repetition). This system, which is highly relevant for the ITER divertor reflectometers, should give broadband probing from 0 to $5 \mathrm{THz}$.

A new $>20 \mathrm{GHz} / \mu$ s ultra-fast sweep reflectometer is being installed on the RFX reverse field pinch device. R.Cavazzana (ENEA, Italy) presented test results of the system, which operates in O-mode with a monostatic antenna. With a $1 \mu$ s sweep period and a quasi-triangular FM waveform (34-39 GHz) the phase and amplitude components could be cleanly separated out of the homodyne signal - i.e. without the need for IQ detection a complex amplitude signal can be extracted with a sample rate equal to the sweep repetition. The frequency sweep introduces a Doppler phase shift, which can be removed by averaging pairs of adjacent up-down sweeps. The phase average gives the pure group delay, while the phase difference gives the pure Doppler shift - which maybe used as a possible error bar estimation. Further, by resampling the reconstructed phase and amplitude signals at times corresponding to particular launch frequencies a series of equivalent fixed frequency signals may be reconstructed, which can then be used for radial correlation measurements.

The possibility of using ultra-fast sweep reflectometry to measure fluctuations was assessed by S.Heuraux (Uni.H.P, Nancy). To follow MHD, islands, sawteeth crashes, Alfvén modes as well as turbulence dynamics (i.e. temporal evolution) and single events such as blobs etc. requires a sufficiently fast sweep that the turbulence is effectively frozen, i.e. a sweep rate faster than the fluctuation correlation time. For Tore Supra conditions a perturbation moving radially at the sound speed would require a sweep rate of around $2 \mathrm{GHz} / \mu \mathrm{s}$. In addition, to track a perturbation poloidally the sweep repetition rate should be much less than the perturbation's transit time across the probing beam. Again for TS conditions, this corresponds to $t_{\text {sweep }}<4 \mu \mathrm{s}$. Such a fast-sweep system is currently under construction for Tore Supra.

\section{Odds and ends}

The final presentation was a report by G.D.Conway (RWG Chair) on the activities of the ITPA specialists working group on reflectometry. On three of the ITER reflectometer systems there has been significant technological as well as design development. The front-end (antenna and transmission line) design of both the high field side and plasma position reflectometers are well progressed. Optimization is still on-going, but no significant problems are expected. The low field side main reflectometer package was recently reassessed by the US party and new antenna layouts have been proposed. The final front-end format is still under discussion. On the divertor reflectometer there has unfortunately been little progress.

Overall, the reflectometer community continues to be extremely active and there has been substantial progress and new results in all areas of reflectometry, with the exception perhaps of Microwave Imaging Reflectometry on which there were no reports 
at this meeting.

Finally, a new International Advisory Committee has been constituted for the next workshop, which is planned for spring 2009 and is to be hosted by the Instituto Superior Téchnico in Lisbon, Portugal.

\section{References}

[1] Proceedings of the 8th International Reflectometry Workshop for Fusion Plasma Diagnostics (IRW8) St.Petersburg, Ioffe Physico-Technical Institute, May 2007. http://www.aug.ipp.mpg.de/IRW/IRW8/

[2] Bretz N.L. 1994 Nucl. Fusion 341283

[3] Sánchez J. 1997 ITER EDA Newsletter 6 no.5 p10 (Vienna: IAEA) ISSN 102-5642

[4] Donné A.J.H. and Sánchez-Sanz J. 2002 Nucl. Fusion 42109

[5] Conway G.D. 2006 Nucl. Fusion 46 S665 